\title{
TRAIL-R1 Is a Negative Regulator of Pro-Inflammatory Responses and Modulates Long-Term Sequelae Resulting from Chlamydia trachomatis Infections in Humans
}

\author{
Mufadhal Al-Kuhlani ${ }^{1}$, James Rothchild ${ }^{5}$, Sukumar Pal ${ }^{2}$, Luis M. de la Maza ${ }^{29}$, Sander Ouburg ${ }^{3}$, \\ Servaas A. Morré ${ }^{3,49}$, Deborah Dean ${ }^{5,6,79}$, David M. Ojcius ${ }^{1 * 9}$
}

1 Department of Molecular Cell Biology, and Health Sciences Research Institute, University of California Merced, Merced, California, United States of America, 2 Department of Pathology and Laboratory Medicine, University of California Irvine, Irvine, California, United States of America, $\mathbf{3}$ Laboratory of Immunogenetics, Medical Microbiology and Infection Prevention, Research School V-ICI, VU University Medical Center, Amsterdam, The Netherlands, 4 Institute of Public Health Genomics, Department of Genetics and Cell Biology, Research School GROW, University of Maastricht, Maastricht, The Netherlands, $\mathbf{5}$ Center for Immunobiology and Vaccine Development, Children's Hospital Oakland Research Institute, Oakland, California, United States of America, $\mathbf{6}$ Graduate Program in Bioengineering, University of California, Berkeley and San Francisco, California, United States of America, 7 Department of Medicine, University of California San Francisco, San Francisco, California, United States of America

\begin{abstract}
The immune system eliminates Chlamydia trachomatis infection through inflammation. However, uncontrolled inflammation can enhance pathology. In mice, TNF-related apoptosis-inducing ligand receptor (TRAIL-R), known for its effects on apoptosis, also regulates inflammation. In humans, the four homologues of TRAIL-R had never been investigated for effects on inflammation. Here, we examined whether TRAIL-R regulates inflammation during chlamydial infection. We examined TRAIL-R1 single nucleotide polymorphisms (SNPs) in an Ecuadorian cohort with and without C. trachomatis infections. There was a highly significant association for the TRAIL+626 homozygous mutant GG for infection vs no infection in this population. To confirm the results observed in the human population, primary lung fibroblasts and bone marrowderived macrophages (BMDMs) were isolated from wildtype (WT) and TRAIL-R-deficient mice, and TRAIL-R1 levels in human cervical epithelial cells were depleted by RNA interference. Infection of BMDMs and primary lung fibroblasts with $C$. trachomatis strain $L_{2}$, or the murine pathogen $C$. muridarum, led to higher levels of MIP2 mRNA expression or IL-1 $\beta$ secretion from TRAIL-R-deficient cells than WT cells. Similarly, depletion of TRAIL-R1 expression in human epithelial cells resulted in a higher level of IL-8 mRNA expression and protein secretion during C. trachomatis infection. We conclude that human TRAILR1 SNPs and murine TRAIL-R modulate the innate immune response against chlamydial infection. This is the first evidence that human TRAIL-R1 is a negative regulator of inflammation and plays a role in modulating Chlamydia pathogenesis.
\end{abstract}

Citation: Al-Kuhlani M, Rothchild J, Pal S, de la Maza LM, Ouburg S, et al. (2014) TRAIL-R1 Is a Negative Regulator of Pro-Inflammatory Responses and Modulates Long-Term Sequelae Resulting from Chlamydia trachomatis Infections in Humans. PLoS ONE 9(4): e93939. doi:10.1371/journal.pone.0093939

Editor: Shibo Jiang, Shanghai Medical College, Fudan University, China

Received February 4, 2014; Accepted March 10, 2014; Published April 2, 2014

Copyright: ( $) 2014$ Al-Kuhlani et al. This is an open-access article distributed under the terms of the Creative Commons Attribution License, which permits unrestricted use, distribution, and reproduction in any medium, provided the original author and source are credited.

Funding: This research was supported by Public Health Service grant from the National Institutes of Health R56 Al078419 and a University of California Presidential Chair for support. The funders had no role in study design, data collection and analysis, decision to publish, or preparation of the manuscript.

Competing Interests: David Ojcius and Deborah Dean are editors for PLoS ONE. This does not alter the authors' adherence to PLOS ONE Editorial policies and criteria.

*E-mail: david.ojcius@gmail.com

9 These authors contributed equally to this work.

\section{Introduction}

Chlamydia trachomatis is the leading cause of bacterial sexuallytransmitted diseases (STDs) and the main cause of preventable blindness worldwide_ENREF_1[1]. According to the Centers for Disease Control, there were more than 1.3 million reported cases in the United States in 2010, which corresponds to an increase of $\sim 8 \%$ in comparison to 2008 [2]. The 19 known serovars of $C$. trachomatis are categorized into three disease groups: ocular, urogenital, and the invasive lymphogranuloma venereum (LGV). The latter pathogens include the $\mathrm{L}_{1}, \mathrm{~L}_{2}, \mathrm{~L}_{2 \mathrm{a}}$, and $\mathrm{L}_{3}$ strains that infect the reticuloendothelial system involving predominantly the lymph nodes $[3,4]$.

Infection of epithelial cells by chlamydiae initiates an inflammatory response through ligation of Toll-like receptors (TLRs) and Nod-like receptors $[5,6]$. These receptors are usually expressed by immune cells such as macrophages, dendritic cells and neutrophils, but also mucosal epithelial cells [7-10]. The engagement of TLRs by microbial products of chlamydiae, such as lipopolysaccharide, initiates the TLR signaling cascade $[5,6]$. Once activated, the Toll/IL-1R (TIR) domain of TLR interacts with various adaptors, such as MyD88, which in turn recruits and activates additional adaptor proteins, including the IL-1 receptor-associated kinases (IRAK) and (TNF)-receptor-associated factor 6 (TRAF6) [11-13]. TRAF6 then activates various proteins that ultimately lead to the phosphorylation of inhibitor of kappa B alpha $(\mathrm{I}-\kappa \mathrm{B} \alpha)$, which subsequently undergoes degradation via ubiquitination. The degradation of $\mathrm{I}-\kappa \mathrm{B}$ releases the activated nuclear factor- $\mathrm{\kappa B}$ (NF$\kappa \mathrm{B}$ ), which allows it to translocate into the nucleus and stimulate the expression of pro-inflammatory components, such as interleukin (IL)-8, IL-6, IL-18, IL- $1 \alpha$ and granulocyte-macrophage 
colony-stimulating factor (GM-CSF) that recruit and activate various immune cells [14-17].

Clearance of infection through inflammation is often an efficient process. However, the mechanisms for clearing chlamydial infection varies among individuals whose immune systems, in addition to clearing the infection, can cause chronic inflammation $[18,19]$. Chronic inflammation and tissue damage seen during $C$. trachomatis infections is caused not by the infectious organism, but by the host's immune response to these pathogens. Therefore inflammation needs to be tightly regulated to avoid uncontrolled immune responses.

Negative regulation of inflammation is accomplished at multiple levels throughout the TLR signaling pathways [20,21]. The first level of regulation involves a decrease in the expression of TLR as the presence of soluble TLRs that can compete with the agonist [22]. Soluble forms of TLR2 and TLR4 dampen the host immune response against infection by preventing the activation of TLRmediated signaling $[23,24]$. Other regulators exert their effect within the cytosol, downstream from TLR ligation. The cytosolic regulators target different components of the TLR signaling pathway such as MyD88, IRAK1, TRAF6, and phosphoinositide 3-kinase [25-30].

The transmembrane receptor of TNF-related apoptosis-inducing ligand receptor (TRAIL-R) is a member of the tumor necrosis factor receptor superfamily that lacks a TIR domain [31]. In addition to its well-established role in inducing apoptosis, TRAIL$\mathrm{R}$ has been reported to modulate inflammation of the host cells in response to various pathogens and diseases [32-34]. Four different TRAIL-Rs have been identified in humans (the transmembrane proteins, TRAIL-R1 through -R4, and a soluble osteoprotegrin) and one full-length receptor in mice [35,36] (Figure 1). TRAIL-R1 and TRAIL-R2, also known as Death Receptor (DR)-4 and DR-5, are the only known receptors that are capable of selectively killing transformed cells but not normal cells, while TRAIL-R3 and TRAIL-R4 serve as decoys [37,38]. TRAIL-R-deficient mice develop normal populations of immune cells $[33,39,40]$, but challenge of these mice with different pathogens and stimuli for TLR2, TLR3 and TLR4 results in enhanced ability of the innate immune system to clear the infection and increased production of different pro-inflammatory-cytokines such as IFN- $\beta$, compared to wildtype mice [33]. The ability of TRAIL-R to downregulate TLR signaling seems to be through decrease activation of $\mathrm{NF}-\kappa \mathrm{B}$ by stabilizing the I- $\kappa \mathrm{B} \alpha$ subunit [33].

While murine TRAIL-R is established as a negative regulator of inflammation, and some progress has been made in understanding the role of TRAIL-R1 in the immune response in humans, nothing is known about the potential role of TRAIL-R1 as a negative regulator of inflammation in humans.

Clear differences in the clinical course of C. trachomatis infections in women have been observed, which are not explained by bacterial factors. This suggests that there might be differences in the host genetic background associated with the recognition of pathogens and the immune response that ensues. Indeed, recent research indicates the relevance for studying the host genetic background in relation to disease susceptibility. In one study, an analysis of the lymphoproliferative responses to $C$. trachomatis antigen among Gambian monozygotic versus dizygotic twins in a trachoma endemic region indicated an almost $40 \%$ contribution of heritability to the observed differences in the response [41].

The effect of single nucleotide polymorphisms (SNPs) on the function of TRAIL-R1 have not been studied yet in C. trachomatis infections. Four TRAIL-R1 SNPs have an effect on the expression of the protein demonstrated as upregulation in silico in human populations based on data in HapMap (http://www.hapmap.org/), but their ability to enhance or diminish inflammation among populations at risk for $C$. trachomatis infection has never been examined.

In this study, we investigate the effect of TRAIL-R1 SNPs in regulating susceptibility to $C$. trachomatis infection among women from a population at risk for STDs, identify TRAIL-R1 as a downregulator of inflammation in human cells, and elucidate the role of murine TRAIL-R in the inflammatory response against chlamydial infection in vitro.

\section{Results and Discussion}

\section{Association of the TRAIL-R1 SNPs with C. trachomatis infection in a human population}

A well-defined Ecuadorian cohort with and without $\boldsymbol{C}$. trachomatis infections was tested for the four known TRAILR1 through -R4 SNPs that could be associated with pathology due to infection.

The human population consisted of 760 women who enrolled in the study. Their ages ranged from 18 to 55 years; the mean age was 29 years. Nine women were excluded from the analyses due to insufficient data. The prevalence of $C$. trachomatis was $16.7 \%$ (126 women); there were no patients with human immunodeficiency virus (HIV-1) infection, and 3.2\% (4 women) were coinfected with Neisseria gonorrhoeae. Table 1 shows the risk factors for C. trachomatis infection. As expected, women younger than 24 years were at increased risk for C. trachomatis infection $(p<0.0003$; OR 2.3807; CI 1.4935 to 3.7950 ), as were women with onset of sexual activity during adolescence. There were no significant differences for infection associated with number of sex partners or prior history of STDs. In addition, the diversity of ompA genotypes for the 126 women included $\mathrm{Ba}$ (6 women), D (19), E (29), F (25), I (5), J (13), Ja (10), L2 (9), L2b (7), and L3 (3). There were no differences among risk groups for infecting genotype.

The distribution of TRAIL-R1 haplotypes differed between women with and without C. trachomatis infection (Table 2). The TRAIL-R1+626 homozygous mutant GG was found to be significantly associated with $C$. trachomatis infection versus no infection ( $p<0.0001$; OR: 5.3 , CI: 2.3763 to 11.8553$)$. There were no synergistic effects for any combination of SNPs. There was no association of any ompA genotype with this SNP.

We have previously shown the association of host genetic factors (genes encoding TLR-2, TLR-4 and TLR-9) with the susceptibility and course of C. trachomatis infection [42-44]. Recently, two other studies $[45,46]$ showed a role for TLR SNPs in the prevalence of pelvic inflammatory disease (PID) among African American women. As TRAIL-R1 can downregulate TLR signaling through decreased activation of NF- $\kappa \mathrm{B}$, our current findings underline the importance of TRAIL-R1 and TLR signaling in the defence against $C$. trachomatis infection. This is consistent with the upregulation of the TRAIL-R1 SNPs in silico.

\section{MIP-2 expression and IL-1 $\beta$ secretion in TRAIL-R-deficient lung fibroblasts}

Most previous studies have focused on the ability of TRAIL-R to induce apoptosis, although some studies also report on the effect of TRAIL-R on infection in animal models [47]. A role for TRAIL-R as a downregulator of TLR-mediated signaling has been reported only for the murine TRAIL-R [33]. In this study, the potential role of TRAIL-R in regulating inflammation during chlamydial infection was examined by isolating primary lung fibroblasts from WT and TRAIL-R-deficient mice and infecting them with G. muridarum (MoPn) and C. trachomatis $\left(\mathrm{LGV} / \mathrm{L}_{2}\right)$. MoPn is an appropriate strain for infecting lung 


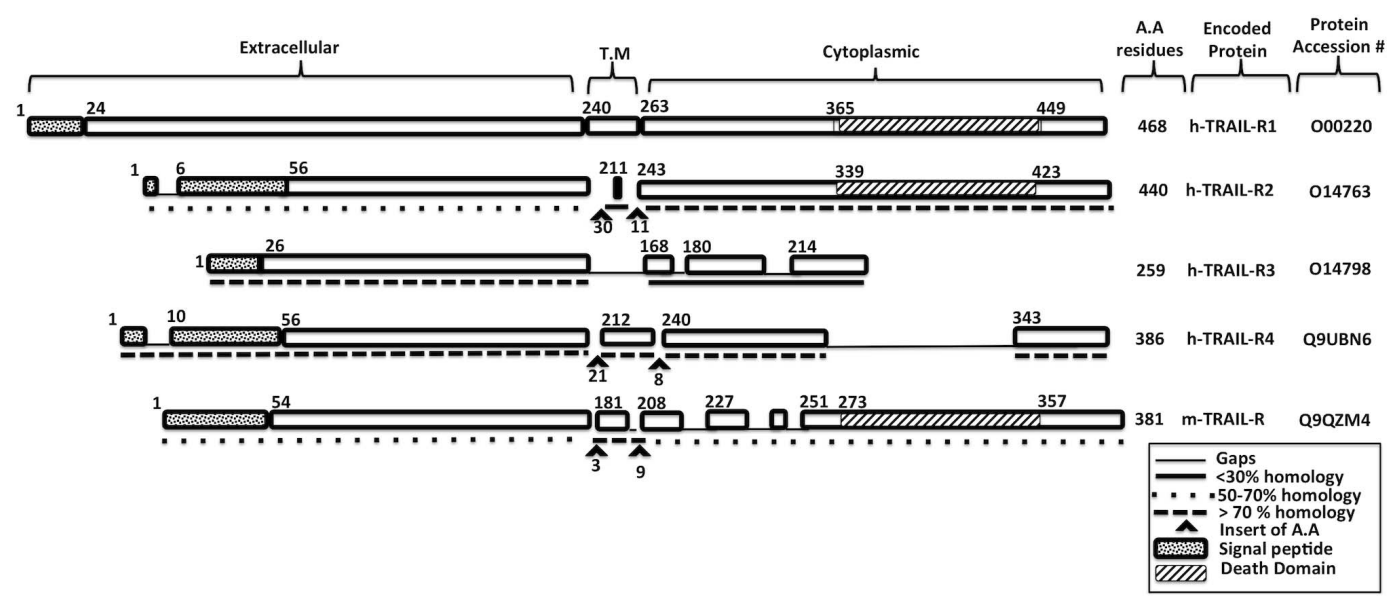

Figure 1. Multiple sequence alignment of human and murine TRAIL-Rs. Sequence alignment shows all four human TRAIL-Rs (hTRAIL-R) and murine TRAIL-R (mTRAIL-R). FASTA files were obtained from the Uniport database at (http://www.uniprot.org). Amino acid (AA) sequences of hTRAILR2-4 and mTRAIL-R were aligned in comparison to hTRAIL-R1 using the ClustalW algorithm. The percentage of homology, insertion or loss of AA sequences, and the unique features of the different receptors are presented with different patterns as indicated. doi:10.1371/journal.pone.0093939.g001

fibroblasts since it causes pneumonitis in mice. As it has not been possible until now to culture primary cervical epithelial cells from mice, lung fibroblasts were also used to characterize the cellular response to the $\mathrm{LGV}$ strain of $C$. trachomatis (L2), which is known to induce a stronger response in the infected cells [48],[49,50]. Since C. muridarum is closely related to the $C$. trachomatis human strains, it has been widely used to study the pathogenesis of chlamydial disease in the reproductive and respiratory tracts of the mouse model [49,50]. As shown in Figure 2A, TRAIL-R-deficient fibroblasts express macrophage inflammatory protein-2 (MIP-2, a murine homologue of human IL-8) at higher levels in response to both $C$. muridarum and $C$. trachomatis infection than WT fibroblasts. However, the secretion of the cytokine IL-1 $\beta$ in response to $C$. muridarum infection was minimal with no significant difference between the WT and TRAIL-R-deficient fibroblasts (Figure 2B).
On the other hand, infection of the fibroblasts with the $\mathrm{L}_{2}$ strain of C. trachomatis induced the secretion of IL-1 $\beta$ more than 60 -fold, compared with uninfected cells (Figure 2A). Similarly to the increased MIP-2 expression during C. trachomatis infection, TRAIL-R-deficient fibroblasts infected with $C$. trachomatis secrete higher levels of IL-1 $\beta$ than the WT cells (Figure 2B).

Even though MIP-2 expression but not IL-1 $\beta$ secretion is stimulated in response to $C$. muridarum infection, $C$. trachomatis infection led to production of both pro-inflammatory mediators, and their levels were enhanced in the absence of TRAIL-R.

\section{Expression of pro-inflammatory mediators in TRAIL-R- deficient macrophages}

Epithelial cells are the preferred host cells for most chlamydial species, but macrophages can also be infected, or at least

Table 1. Risk of $C$. trachomatis infection in humans by age group*.

\begin{tabular}{|c|c|c|c|c|c|c|c|c|c|}
\hline \multirow[t]{2}{*}{ Risk } & \multicolumn{2}{|c|}{$18-23$ yrs $(n=251)$} & \multirow[t]{2}{*}{$P$ value } & \multicolumn{2}{|c|}{$24-29$ yrs $(n=250)$} & \multirow[t]{2}{*}{$P$ value } & \multicolumn{2}{|c|}{$>29$ yrs $(n=250)$} & \multirow[t]{2}{*}{ P value } \\
\hline & $\mathrm{CT}+$ & CT- & & CT+ & CT- & & $\mathrm{CT}+$ & CT- & \\
\hline $\operatorname{Age}^{* *}$ & 65 & 186 & & 32 & 218 & & 27 & 223 & $p<0.0003$ \\
\hline \multicolumn{10}{|c|}{ Onset sexual activity: } \\
\hline adolescence & 65 & 112 & $P=0.0018$ & 32 & 150 & $p<0.018$ & 27 & 177 & $p<0.0633$ \\
\hline adulthood & 0 & 74 & & 0 & 68 & & 0 & 46 & \\
\hline \multicolumn{10}{|c|}{ No. of sex partners: } \\
\hline 1 & 27 & 99 & NA & 10 & 75 & NA & 10 & 70 & NA \\
\hline$>1$ & 38 & 87 & & 22 & 143 & & 17 & 153 & \\
\hline \multicolumn{10}{|c|}{ Prior history STDs: } \\
\hline Yes & 40 & 100 & NA & 19 & 111 & NA & 17 & 103 & NA \\
\hline No & 25 & 86 & & 13 & 107 & & 10 & 120 & \\
\hline \multicolumn{10}{|c|}{ Co-STD infections: } \\
\hline HIV & 0 & 251 & NA & 0 & 250 & NA & 0 & 250 & NA \\
\hline GC & 2 & 249 & NA & 1 & 249 & NA & 1 & 249 & NA \\
\hline
\end{tabular}

*Nine women were excluded due to missing data.

**Calculated across all age groups.

doi:10.1371/journal.pone.0093939.t001 
Table 2. Distribution of four TRAILR1 SNPs in C. trachomatis positive (CT+) and negative (CT-) women in Ecuador.

\begin{tabular}{|c|c|c|c|c|c|c|c|}
\hline & \multicolumn{3}{|l|}{$\mathbf{C T +}$} & \multicolumn{3}{|l|}{ Ст- } & \multirow[t]{2}{*}{$P$ value } \\
\hline & Wildtype & Heterozygote & Mutant & Wildtype & Heterozygote & Mutant & \\
\hline TRAILR1 +626 C>G & $13.2 \%$ & $40.4 \%$ & $46.4 \%$ & $35.9 \%$ & $39.9 \%$ & $24.2 \%$ & $p<0.0001$ \\
\hline TRAILR1 $+683 \mathrm{~A}>\mathrm{C}$ & $59.2 \%$ & $27.1 \%$ & $13.7 \%$ & $64.2 \%$ & $27.9 \%$ & $7.9 \%$ & NS \\
\hline TRAILR1 +422 G>A & $22.7 \%$ & $51.9 \%$ & $25.4 \%$ & $25.3 \%$ & $50.9 \%$ & $23.8 \%$ & NS \\
\hline TRAILR1 +1387 A>G & $83.0 \%$ & $20.8 \%$ & $6.2 \%$ & $79.1 \%$ & $15.9 \%$ & $5.0 \%$ & NS \\
\hline
\end{tabular}

doi:10.1371/journal.pone.0093939.t002
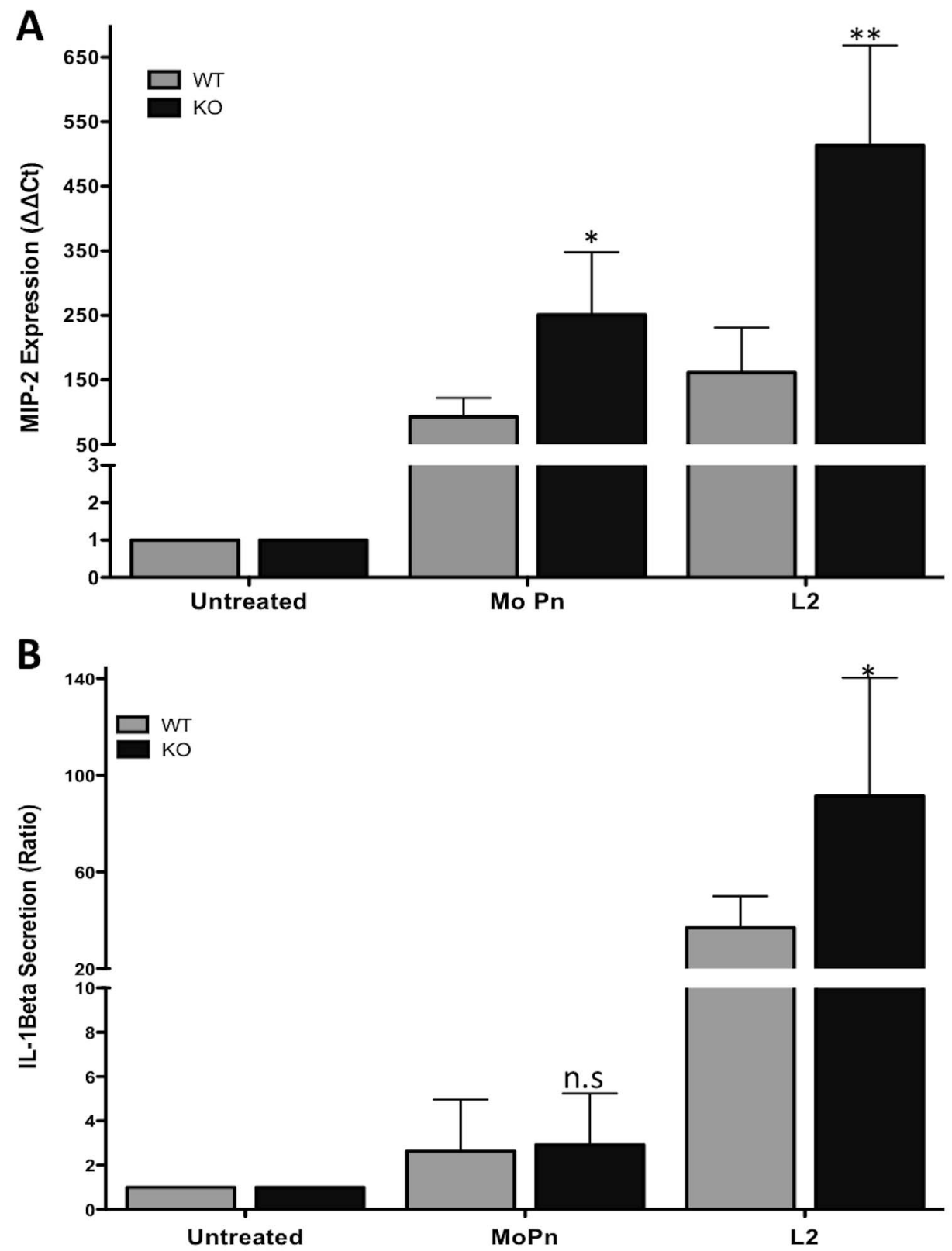

Figure 2. TRAIL-R deficiency increases MIP-2 and IL-1 $\beta$ levels in primary lung fibroblasts in response to a Chlamydia infection. WT and TRAIL-R-deficient cells were infected with either C. muridarum (MoPn) or C. trachomatis (L2) strains at an MOI of 1 for 24 hrs. (A) mRNA levels of MIP-2 was measured via qPCR. Cycle threshold $(\mathrm{Ct})$ values were normalized to GAPDH and relative expression $(\Delta \Delta \mathrm{Ct})$ was calculated compared to uninfected cells. (B) ELISA measurement for IL-1 $\beta$. The ratio of increased IL-1 $\beta$ level was calculated with respect to uninfected cells. Error bars represent standard deviations from 3 separate experiments. ${ }^{*}$ indicates $p<0.05 ;{ }^{* *}$ indicates $p<0.01$, compared to infected WT cells. doi:10.1371/journal.pone.0093939.g002 
stimulated, with Chlamydia [51-53]. We thus isolated bone marrow cells from WT and TRAIL-R-deficient mice and induced their differentiation into bone-marrow derived macrophages (BMDMs). Flow cytometry analysis, shown in Figure S1, confirmed that the great majority of cultured cells expressed the specific markers, CD11b and F4/80. Compared with the uninfected groups, BMDMs infected with C. muridarum expressed low levels of MIP-2 and secreted little IL-1 $\beta$, with no significant difference between WT and TRAIL-R-deficient cells (Figure 3A, B). In contrast, BMDMs infected with C. trachomatis L2 expressed high levels of both MIP-2 and IL-1 $\beta$, which were enhanced by TRAIL-R deficiency (Figure 3A, B).
Expression of TRAIL-R1 through TRAIL-R4 in human cells

With few exceptions, studies of the role of TRAIL-R in regulating inflammation had been performed using the mouse model [33]. We therefore first examined via PCR whether the four human TRAIL-Rs (TRAIL-R1 through TRAIL-R4) are expressed in cells that are commonly used in research on innate immunity and chlamydial infections: the cervical epithelial cell line, HeLa; the human monocyte cell line, THP-1; and the human embryonic kidney cell line, HEK-293. As shown in Figure 4A, both HeLa and HEK293 cells express high levels of all four subtypes of TRAIL-R, with the exception to TRAIL-R3, which is
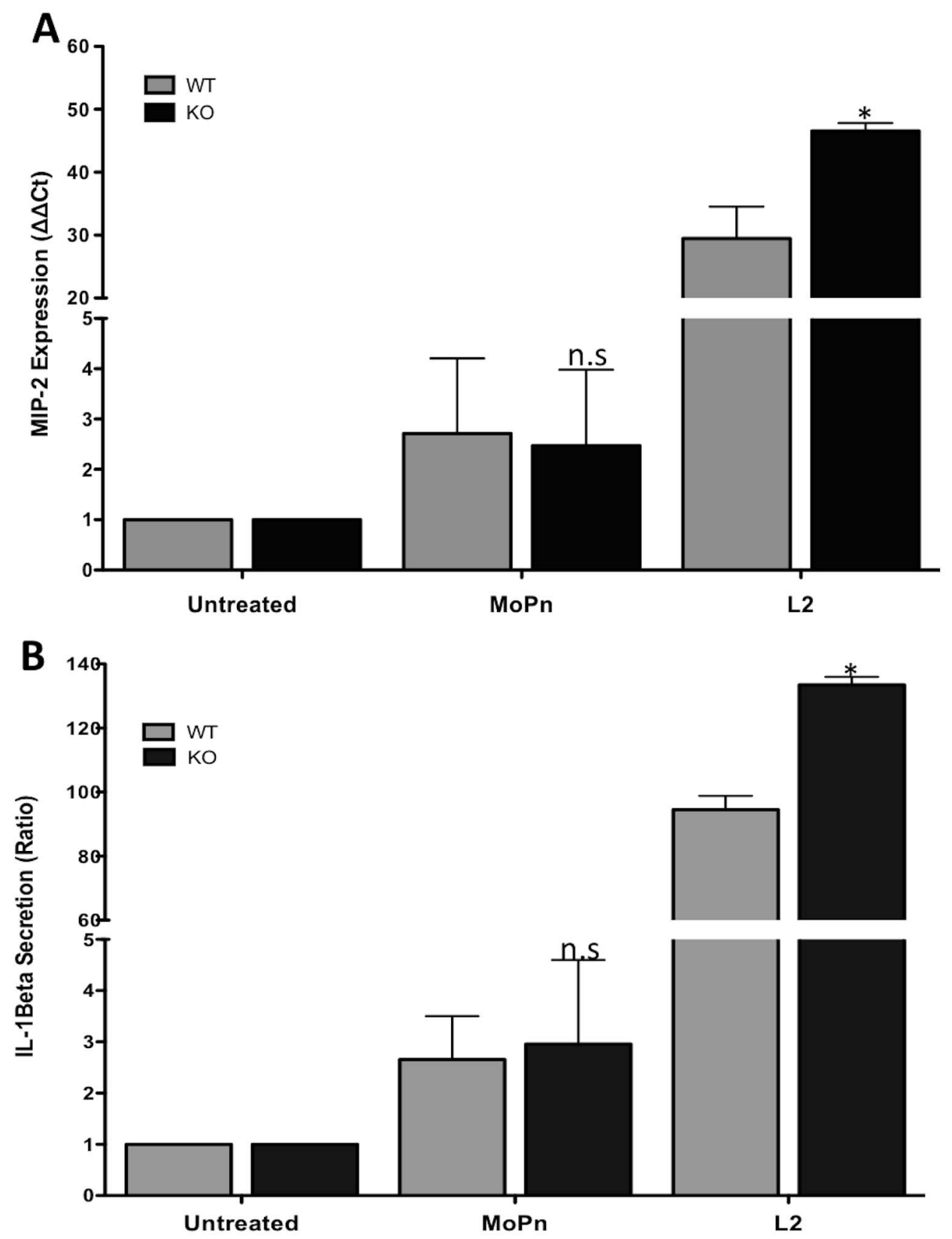

Figure 3. TRAIL-R-deficient BMDMs express higher MIP-2 and IL-1 $\beta$ levels in L2- but not MoPn-infected cells. BMDMs were infected with C. muridarum (MoPn) or C. trachomatis (L2) as for fibroblasts in Figure 2 above. QPCR analysis was performed for MIP-2 (A), and IL-1 $\beta$ secretion was measured by ELISA (B). Error bars represent standard deviations from 3 separate experiments. * indicates $p<0.05$; ${ }^{*}$ indicates $p<0.01$, compared to infected WT cells.

doi:10.1371/journal.pone.0093939.g003 
A

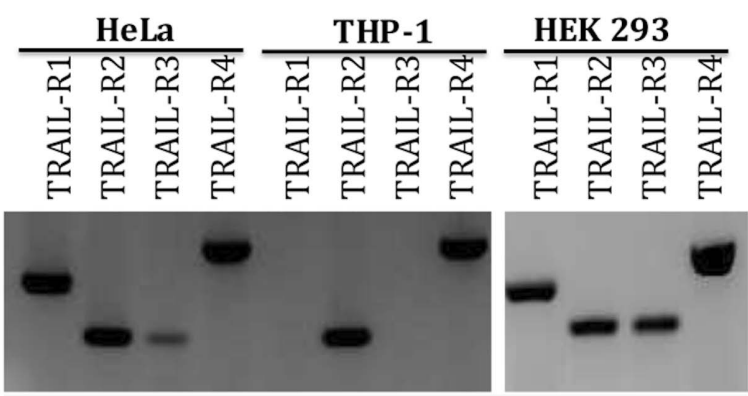

B

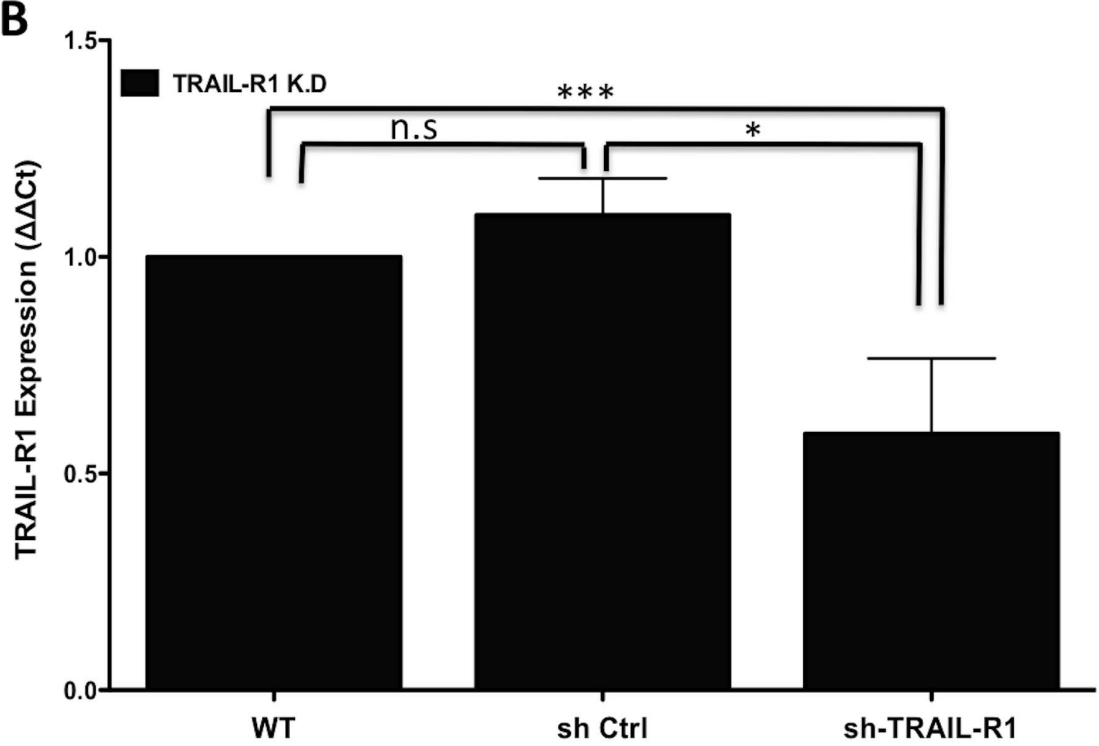

Figure 4. Expression of TRAIL-R1-R4 and depletion of TRAIL-R1 in human cells. (A) A $2 \%$ agarose gel electrophoresis gel shows RT-PCR amplification of TRAIL-R1 (214 bp), TRAIL-R2 (145 bp), TRAIL-R3 (141 bp) and TRAIL-R4 (271 bp) mRNA from HeLa, THP-1 and HEK293 cell lines. (B) HeLa cells transduced with TRAIL-R1 specific shRNA (sh-TRAIL-R1) show a reduced level of TRAIL-R1 mRNA when measured by real-time PCR and compared with wildtype (WT) and non-target control (sh Ctrl). Error bars represent standard deviations from at least 3 independent experiments. $* * *$ indicates $p<0.001(p=0.0152)$ and "n.s" indicates "not significant" $(p=0.1229)$ compared with WT. The $p$ value for sh-TRAIL-R1 compared to nontarget control is 0.0107

doi:10.1371/journal.pone.0093939.g004

barely expressed in HeLa cells; while the THP-1 cell line expressed only TRAIL-R2 and TRAIL-R4.

\section{Expression and secretion of IL-8 in TRAIL-R1-deficient cervical epithelial cells}

The protein structures of both TRAIL-R1 and TRAIL-R2 resemble each other (Figure 1) [54] [55]. We thus chose to focus our initial studies on TRAIL-R1 given its homology to murine TRAIL-R. To evaluate the role of TRAIL-R1 in vitro, specific shRNA lentiviral particles were used to deplete TRAIL-R1 in HeLa cells. As measured by qPCR, there was a significant reduction of TRIAL-R1 mRNA levels in transduced HeLa cells, compared to the WT and non-target shRNA control cells (sh Ctrl) (Figure 4B).

Secretion of the chemokine IL-8, which is an indicator of early stages of the inflammatory response, has been previously described for HeLa cells infected with Chlamydia $[14,17,56]$. The levels of IL8 mRNA expression or IL-8 protein secretion in response to $C$. trachomatis infection in TRAIL-R1-depleted cells were significantly higher than in infected WT or non-target shRNA control cells (Figure 5A, B).

\section{Concluding remarks}

We here show that TRAIL-R can negatively regulate initiation of inflammation in humans and mice during chlamydial infection.

The role of TRAIL-R as a negative regulator of inflammation had been previously addressed in the murine model [33]. The exvivo results presented here demonstrate that TRAIL-R is involved in regulation of pro-inflammatory cytokine production in primary lung fibroblasts in response to $C$. trachomatis or $C$. muridarum infection. However, infection with $C$. trachomatis, but not $C$. muridarum, is capable of inducing such responses in the BMDMs. The different responses to $C$. muridarum infection observed between lung fibroblasts and BMDMs, and between C. trachomatis and $C$. muridarum infection of BMDMs, suggest that the mechanisms of negative regulation of inflammation by TRAIL-R may depend on the tissues being infected or the specific pathogen strain. In fact, the L2 strain of C. trachomatis used in these studies is a pathogen of the reticuloendothelial system involving predominantly the lymph nodes, whereas C. muridarum is not.

This is the first report that human TRAIL-R1 also functions as a negative regulator of inflammation. The exact mechanisms used by TRAIL-R 1 to dampen pro-inflammatory responses in humans remains to be investigated, but could provide potential biomarkers indicative for the course of infection. The link between TLR 


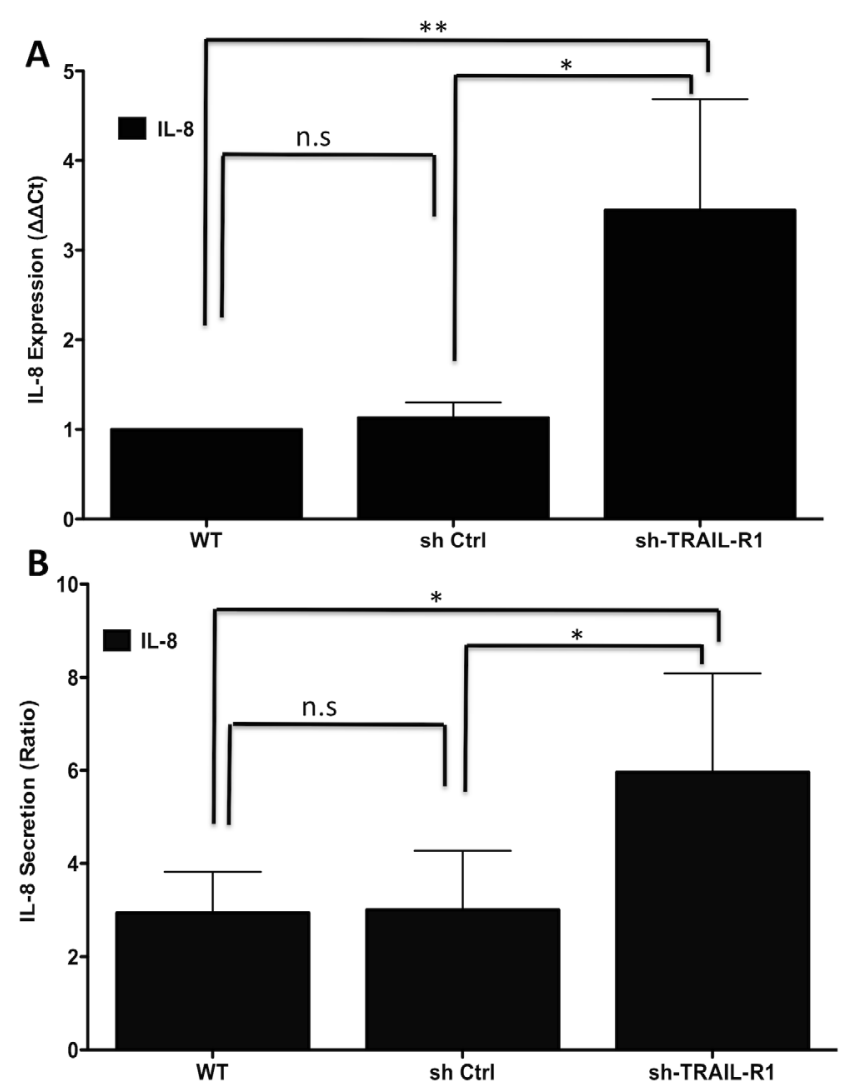

Figure 5. Increased IL-8 expression and secretion in TRAIL-R1depleted cervical epithelial cells. HeLa cells were infected with $C$. trachomatis strain (L2) at an MOI of 1. (A) RNA was isolated $24 \mathrm{hpi}$ for quantification of IL-8 expression using qPCR. The data were normalized to GAPDH, and infected wild type HeLa cells were used to calculate the relative expression $(\Delta \Delta \mathrm{Ct})$. Error bars represent standard deviations from 4 separate experiments. ** indicates $p=0.0075$; and "n.s." indicates "not significant" $(p=0.1736)$, compared with infected WT cells. The $p$ value for sh-TRAIL-R1 compared with non-target control is 0.01. (B) ELISA measurement of IL-8 secretion. Error bars represent standard deviations from 4 separate experiments. ${ }^{*}$ indicates $p<0.05$; $(p=0.0394)$; and "n.s." indicates "not significant" $(p=0.9332)$, compared with infected WT cells. The $p$ value for sh-TRAIL-R1 compared to nontarget control is 0.0479 .

doi:10.1371/journal.pone.0093939.g005

signaling and apoptosis has been reported in various studies where proteins that are known to facilitate apoptosis, such as Nur77, PKR or TRAIL, are up-regulated due to activation of some TLR pathways [57-62]. Since TRAIL-R1 is a trigger of apoptosis via the adaptor domain, Fas-associated death domain (FADD) and caspase-8, and C. trachomatis modulates host cell death during infection, evaluating the effect of TRAILR-R1 on the activation of the apoptotic pathway during chlamydial infection may contribute to explaining the mechanism of action of TRAIL-R1 as a negative regulator. Indeed, the pattern of low mRNA expression of TRAIL-R1 at early times of infection and the increase at later stages (data not shown) could be related to the time-course of $C$. trachomatis infection on apoptosis of the infected cells [34,63]. Another indicator for the possible involvement of FADD in the function of TRAIL-R as a negative regulator is the observation that FADD can modulate the activity of NF- $\kappa$ B and IL- $1 \beta$ with a pattern that is similar to TRAIL-R [64-66]. In addition, the role that the truncated receptors, TRAIL-R3 and TRAIL-R4, may play during inflammation has never been addressed. It is tempting to speculate that, behaving as dominant negative versions of TRAIL-R1, the truncated receptors may have the opposite effect of TRAIL-R1, enhancing rather than dampening inflammation. It is possible that the absence of TRAIL-R results in a decrease of apoptosis in the inflamed tissue and, therefore, increase the intensity of the inflammatory response. Characterization of this gene family may help to gain a better understanding of their role in Chlamydia pathogenesis and could lead to the development of therapeutic approaches to minimize the side effects of inflammation when infection occurs.

\section{Methods and Materials}

\section{Human study populations}

Female commercial sex workers $(n=760)$ ranging in age from 18-55 years were recruited and enrolled in the study through health care clinics in Quito, Ecuador, from August 2002 to August 2005. Potential participants were excluded if they had taken antibiotics within the four weeks preceding study enrollment, had a history of any cancer diagnosis or collagen vascular disease, or were unwilling to complete the study questionnaire and provide biological samples. Written informed consent was obtained from all participants, and approval for the study was granted by the Institutional Review Boards (IRBs) of Children's Hospital Oakland Research Institute and La Pontificia Universidad Católica del Ecuador, Quito, Ecuador, according to the tenets of the Declaration of Helsinki.

All study participants were administered survey questionnaires about risk factors for HIV and other sexually transmitted infections. Participants were excluded from the final analyses if either their C. trachomatis diagnostic status or SNP data were missing.

\section{C. trachomatis detection and ompA genotyping in patient populations}

Cervical swab samples were collected from the study participants using standard techniques and the presence of C. trachomatis was determined and commercial nucleic acid amplification tests (NAAT) (Amplicor; Roche Molecular Systems) [67]. Indeterminate samples were resolved using an in-house PCR as previously described [67].

All samples from the Ecuadorian population that were positive by Amplicor and/or in-house PCR were subjected to ompA genotyping as described elsewhere [68].

\section{Haplotype determination}

In order to determine the homogeneity or heterogeneity of the Ecuadorian population, the four SNPs for TRAIL-R1 were analyzed. The haplotypes were inferred using PHASE v2.1.1 [69] and SNPHAP [70].

\section{SNP detection}

Genomic DNA was extracted from cryopreserved blood samples using either the DNeasy Blood \& Tissue Kit (Qiagen, Valencia, CA) or the MagNaPure LG isolator (Roche Molecular Biochemicals, Germany) according to the manufacturer's instructions. Both techniques provided enough DNA for reproducible genetic analyses. For the Ecuador population, single nucleotide polymorphisms (SNPs) in amplified DNA fragments were identified by the Sequenom MassARRAY system that uses MALDITOF Mass Spectrometry. The assays were run at the W.M. Keck Foundation Biotechnology Resource Laboratory at Yale University, New Haven, Connecticut. Custom software was used to design SNP assays and analyze the data. Different size primer 
extension products were generated for the two alleles of a given SNP. The presence of one or both alleles is scored by the software for each sample. The assays were designed to interrogate four SNPS per sample. These included TRAIL-R1 SNPs +422 G>A (141 His $\rightarrow$ Arg; rs6557634); +626 C > G (209 Arg>Thr; rs20575); +683 A>C (228 Glu>Ala; rs20576); and +1387 A>G (441 Lys $>$ Arg; rs2230229).

\section{Statistical analyses}

Statistical analyses for the human data were performed as previously described [71]. Briefly, the chi-square test was used for determining frequency of SNP comparisons, the combined effect of the SNPs and risk factors between the groups. Each SNP was tested for Hardy-Weinberg equilibrium. Multivariate logistic regression was used for analysis of STD risk factors, SNPs and SNP interactions. Logic regression was also performed to identify interactions among the SNPs as previously described [71]. Fisher's exact or $\chi^{2}$ tests were also used to compare the SNP genotypes between $C$. trachomatis positive and negative STD patients. STATA 10 was used for analyses (Texas). $p$ values $<0.05$ were considered statistically significant.

The statistical significance for the ex vivo and in vitro experiments was evaluated using GraphPad Instat software (GraphPad Software Inc, La Jolla, CA) by unpaired Student's ttest. A value of $p<0.05$ was considered significant. Data are presented as the cumulative result of at least 3 independent experiments, unless stated otherwise.

\section{Chlamydia stocks}

The $\mathrm{L}_{2}$ serovar (434 strain) of C. trachomatis and C. muridarum (previously called $C$. trachomatis mouse pneumonitis $(\mathrm{MoPn})$, strain Nigg II) were grown in HeLa cells as described before [72,73]. The multiplicity of infection (MOI) for $C$. trachomatis and $C$. muridarum was determined by infecting HeLa cell monolayer cultures as previously described [73].

\section{Eukaryotic cells}

The human cervical carcinoma cell line, HeLa 229, was obtained from American Type Culture Collection (ATCG). The THP1 monocyte cell lines was previously described [74].

To isolate murine cells, TRAIL-R-deficient mice in the C57Bl/ 6 background were obtained from Dr. Astar Winoto (University of California, Berkeley) [33] and were housed at the University of California, Irvine, Vivarium. The University of California, Irvine, Animal Care and Use Committee approved all animal protocols. The mice were euthanized with an overdose of xylazine plus ketamine. To produce BMDMs, femurs from two WT and TRAIL-R-deficient mice were cleaned from tissues and briefly sterilized in $70 \%$ ethanol. BMDMs were prepared as described elsewhere [33]. BMDM cells were harvested using trypsin and the cell populations were analyzed by flow cytometry for the expression of BMDM specific receptor using anti-mouse CD11bPE and F4/80-APG (eBiosciences). Flow cytometry data analysis was performed using FlowJo software, version 7.6.1 (TreeStar).

Primary lung fibroblasts were prepared from wildtype and TRAIL-R-deficient mice, following the procedures previously described [75,76]. Fourteen days post isolation, the primary lung fibroblasts were seeded into 12 well plates for infection assays, as we previously described [77].

\section{Generation of TRAIL-R1-depleted HeLa cells using lentiviral shRNA}

HeLa cells were seeded in 96-well plates and then transduced in the presence of $8 \mu \mathrm{g} / \mathrm{ml}$ Polybrene for $48 \mathrm{hrs}$ with shRNATRAIL-R1 specific lentiviral particles purchased from SigmaAldrich (catalog number NM?003842) at a multiplicity of infection (MOI) of 1. Non-target shRNA control cells were also generated using an irrelevant sequence (Sigma, SHC002 V). Cells successfully transduced with lentiviral particles were selected by the addition of media containing $2.5 \mu \mathrm{g} / \mathrm{ml}$ puromycin (SigmaAldrich). The knockdown efficiency of TRAIL-R1 was verified by q-PCR analysis.

\section{Ex vivo and in vitro cell culture and infection}

Sixteen to twenty four hours prior to infection, $1 \times 10^{6} /$ well of murine BMDMs, $1 \times 10^{6}$ murine lung fibroblasts/well, or $5 \times 10^{4}$ cells/well of HeLa cells were plated on 24 well plates in antibiotic free culture media. Cells growing to about $60 \%$ confluency were infected with either C. trachomatis (LGV/L2) or C. muridarum $(\mathrm{MoPn})$ as indicated at an MOI of 1.0 and incubated at $37^{\circ} \mathrm{C}$ with $5 \% \mathrm{CO}_{2}$. Twenty four hours post infection (hpi), supernatants were collected for ELISA analysis and infected cells were lysed in RLT buffer for RNA isolation.

\section{Reverse transcription and PCR (RT-PCR)}

Total RNA were isolated from cells using the Qiagen RNeasy kit (Qiagen) following the manufacturer's instructions. The synthesis of the complementary DNA (cDNA) templates were conducted according to the manufacturer's instructions (TaqMan, Roch). The PCR was performed using Qiagen Fast Cycling PGR Kit. PGR conditions included denaturation steps at $95^{\circ} \mathrm{C}$ for $5 \mathrm{~min}$, followed by 35 cycles at $96^{\circ} \mathrm{C}$ for $5 \mathrm{sec}, 60^{\circ} \mathrm{C}$ for $5 \mathrm{sec}$ and $68^{\circ} \mathrm{C}$ for $5 \mathrm{sec}$ followed by $72^{\circ} \mathrm{C}$ for $1 \mathrm{~min}$. The PCR products were separated by $2 \%$ agarose gel electrophoresis and visualized using ethidium bromide. The primers are listed in Table S1.

\section{Quantitative-PCR (qPCR)}

Total RNA and cDNA were generated as explained above. The q-PCR analysis was conducted in triplicates of $20 \mu$ final volume using Mx3000P (Stratagene, La Jolla, CA) with Brilliant III Ultra Fast STBR Green qPCR master mix (Stratagene). Negative controls include a no-RT control and no cDNA template control $\left(\mathrm{H}_{2} \mathrm{O}\right.$ alone). Real-time PCR included initial denaturation at $95^{\circ} \mathrm{C}$ for $3 \mathrm{~min}$, followed by 40 cycles at $95^{\circ} \mathrm{C}$ for $5 \mathrm{sec}, 60^{\circ} \mathrm{C}$ for $20 \mathrm{sec}$, and one cycle at $95^{\circ} \mathrm{C}$ for $1 \mathrm{~min}, 55^{\circ} \mathrm{C}$ for $30 \mathrm{sec}$, and $95^{\circ} \mathrm{C}$ for $30 \mathrm{sec}$. The averages of the collected data were normalized to the activity of a house-keeping gene, GAPDH. For mouse BMDM and lung fibroblasts, the relative expressions $(\Delta \Delta \mathrm{Ct})$ were calculated using uninfected cells (WT or TRAIL-R deficient) as a baseline. For the human cell line, infected wildtype HeLa cells were used as a baseline for the $\Delta \Delta \mathrm{Ct}$ calculation.

\section{ELISA for cytokine secretion}

The mouse IL-1 $\beta$ ELISA kit (eBioscience Cat\# 88-7013-76) and human CXCL8/IL-8 cytokine ELISA kit (R\&D Systems, DY208) were used according to manufacturer's instructions. Ratios of the secreted IL-1 $\beta$ and IL-8 were calculated in comparison to the untreated samples.

\section{Supporting Information}

Figure S1 Representative flow cytometry dot plot of differentiated BMDM cells. Cultured bone marrow cells were stained with 
BMDM-specific antibodies against mouse CD1 lb-PE and F4/80APC, seven days post-isolation.

(TIFF)

Table S1 List of primers used for qPCR.

(TIFF)

\section{References}

1. Miller WC, Ford CA, Morris M, Handcock MS, Schmitz JL, et al. (2004) Prevalence of chlamydial and gonococcal infections among young adults in the United States. JAMA 291: 2229-2236.

2. Prevention (2010) Sexually Transmitted Disease Surveillance. In: CDC, editor. CDC.

3. Gomes JP, Bruno WJ, Nunes A, Santos N, Florindo C, et al. (2007) Evolution of Chlamydia trachomatis diversity occurs by widespread interstrain recombination involving hotspots. Genome research 17: 50-60.

4. Herring A, Richens J (2006) Lymphogranuloma venereum. Sexually transmitted infections 82 Suppl 4: iv23-25.

5. Joyee AG, Yang X (2008) Role of toll-like receptors in immune responses to chlamydial infections. Curr Pharm Des 14: 593-600.

6. Shimada K, Crother TR, Arditi M (2012) Innate immune responses to Chlamydia pneumoniae infection: role of TLRs, NLRs, and the inflammasome. Microbes Infect 14: 1301-1307.

7. Lemaitre B, Nicolas E, Michaut L, Reichhart JM, Hoffmann JA (1996) The dorsoventral regulatory gene cassette spatzle/Toll/cactus controls the potent antifungal response in Drosophila adults. Cell 86: 973-983.

8. Poltorak A, He X, Smirnova I, Liu MY, Van Huffel C, et al. (1998) Defective LPS signaling in $\mathrm{C} 3 \mathrm{H} / \mathrm{HeJ}$ and $\mathrm{C} 57 \mathrm{BL} / 10 \mathrm{ScCr}$ mice: mutations in Tlr4 gene. Science 282: 2085-2088.

9. Meissner TB, Li A, Kobayashi KS (2012) NLRC5: a newly discovered MHC class I transactivator (CITA). Microbes Infect 14: 477-484.

10. Said-Sadier N, Ojcius DM (2012) Alarmins, inflammasomes and immunity. Biomed J 35: 437-449.

11. Medzhitov R, Preston-Hurlburt P, Kopp E, Stadlen A, Chen C, et al. (1998) MyD88 is an adaptor protein in the hToll/IL-1 receptor family signaling pathways. Mol Cell 2: 253-258.

12. Cao Z, XiongJ, Takeuchi M, Kurama T, Goeddel DV (1996) TRAF6 is a signal transducer for interleukin-1. Nature 383: 443-446.

13. Cao Z, Henzel WJ, Gao X (1996) IRAK: a kinase associated with the interleukin-1 receptor. Science 271: 1128-1131.

14. Buchholz KR, Stephens RS (2006) Activation of the host cell proinflammatory interleukin-8 response by Chlamydia trachomatis. Cell Microbiol 8: 1768-1779.

15. Dessus-Babus S, Knight ST, Wyrick PB (2000) Chlamydial infection of polarized HeLa cells induces PMN chemotaxis but the cytokine profile varies between disseminating and non-disseminating strains. Cell Microbiol 2: 317-327.

16. Lu H, Shen G, Brunham RC (2000) Chlamydia trachomatis infection of epithelial cells induces the activation of caspase-1 and release of mature IL-18. Journal of immunology 165: 1463-1469.

17. Rasmussen SJ, Eckmann L, Quayle AJ, Shen L, Zhang YX, et al. (1997) Secretion of proinflammatory cytokines by epithelial cells in response to Chlamydia infection suggests a central role for epithelial cells in chlamydial pathogenesis. The Journal of clinical investigation 99: 77-87.

18. Campbell LA, Patton DL, Moore DE, Cappuccio AL, Mueller BA, et al. (1993) Detection of Chlamydia trachomatis deoxyribonucleic acid in women with tubal infertility. Fertil Steril 59: 45-50.

19. Kiviat NB, Wolner-Hanssen P, Peterson M, Wasserheit J, Stamm WE, et al. (1986) Localization of Chlamydia trachomatis infection by direct immunofluorescence and culture in pelvic inflammatory disease. Am J Obstet Gynecol 154: 865-873.

20. Coll RC, O'Neill LA (2010) New insights into the regulation of signalling by tolllike receptors and nod-like receptors. J Innate Immun 2: 406-421.

21. Richards KH, Macdonald A (2011) Putting the brakes on the anti-viral response: negative regulators of type I interferon (IFN) production. Microbes Infect 13: 291-302.

22. McCartney-Francis N, Jin W, Wahl SM (2004) Aberrant Toll receptor expression and endotoxin hypersensitivity in mice lacking a functional TGFbeta 1 signaling pathway. Journal of immunology 172: 3814-3821.

23. Iwami KI, Matsuguchi T, Masuda A, Kikuchi T, Musikacharoen T, et al. (2000) Cutting edge: naturally occurring soluble form of mouse Toll-like receptor 4 inhibits lipopolysaccharide signaling. Journal of immunology 165: 6682-6686.

24. LeBouder E, Rey-Nores JE, Rushmere NK, Grigorov M, Lawn SD, et al. (2003) Soluble forms of Toll-like receptor (TLR)2 capable of modulating TLR2 signaling are present in human plasma and breast milk. Journal of immunology 171: 6680-6689.

25. Janssens S, Burns K, Tschopp J, Beyaert R (2002) Regulation of interleukin-1and lipopolysaccharide-induced NF-kappaB activation by alternative splicing of MyD88. Current biology: CB 12: 467-471.

26. Burns K, Janssens S, Brissoni B, Olivos N, Beyaert R, et al. (2003) Inhibition of interleukin 1 receptor/Toll-like receptor signaling through the alternatively spliced, short form of MyD88 is due to its failure to recruit IRAK-4. J Exp Med 197: 263-268.

\section{Author Contributions}

Conceived and designed the experiments: LMM SAM DD DMO. Performed the experiments: MAK SP. Analyzed the data: MAK JR SP LMM SO SAM DD DMO. Contributed reagents/materials/analysis tools: JR LMM SAM DMO. Wrote the paper: MAK LMM DD DMO.

27. Kinjyo I, Hanada T, Inagaki-Ohara K, Mori H, Aki D, et al. (2002) SOCS1/ $\mathrm{JAB}$ is a negative regulator of LPS-induced macrophage activation. Immunity 17: 583-591.

28. Kobayashi K, Hernandez LD, Galan JE, Janeway CA Jr, Medzhitov R, et al. (2002) IRAK-M is a negative regulator of Toll-like receptor signaling. Cell 110 : $191-202$.

29. Fukao T, Tanabe M, Terauchi Y, Ota T, Matsuda S, et al. (2002) PI3Kmediated negative feedback regulation of IL-12 production in DCs. Nat Immunol 3: 875-881.

30. Boone DL, Turer EE, Lee EG, Ahmad RC, Wheeler MT, et al. (2004) The ubiquitin-modifying enzyme A20 is required for termination of Toll-like receptor responses. Nat Immunol 5: 1052-1060.

31. Wu GS, Burns TF, Zhan Y, Alnemri ES, El-Deiry WS (1999) Molecular cloning and functional analysis of the mouse homologue of the KILLER/DR5 tumor necrosis factor-related apoptosis-inducing ligand (TRAIL) death receptor. Cancer Res 59: 2770-2775.

32. Bodmer JL, Holler N, Reynard S, Vinciguerra P, Schneider P, et al. (2000) TRAIL receptor-2 signals apoptosis through FADD and caspase-8. Nature cell biology 2: 241-243.

33. Diehl GE, Yue HH, Hsieh K, Kuang AA, Ho M, et al. (2004) TRAIL-R as a negative regulator of innate immune cell responses. Immunity 21: 877-889.

34. Kuang AA, Diehl GE, Zhang J, Winoto A (2000) FADD is required for DR4and DR5-mediated apoptosis: lack of trail-induced apoptosis in FADD-deficient mouse embryonic fibroblasts. J Biol Chem 275: 25065-25068.

35. Pitti RM, Marsters SA, Ruppert S, Donahue CJ, Moore A, et al. (1996) Induction of apoptosis by Apo-2 ligand, a new member of the tumor necrosis factor cytokine family. J Biol Chem 271: 12687-12690.

36. Wiley SR, Schooley K, Smolak PJ, Din WS, Huang CP, et al. (1995) Identification and characterization of a new member of the TNF family that induces apoptosis. Immunity 3: 673-682.

37. Ashkenazi A, Holland P, Eckhardt SG (2008) Ligand-based targeting of apoptosis in cancer: the potential of recombinant human apoptosis ligand 2/ Tumor necrosis factor-related apoptosis-inducing ligand (rhApo2L/TRAIL). J Clin Oncol 26: 3621-3630.

38. Wang S (2008) The promise of cancer therapeutics targeting the TNF-related apoptosis-inducing ligand and TRAIL receptor pathway. Oncogene 27: 62076215 .

39. Cretney E, Uldrich AP, Berzins SP, Strasser A, Godfrey DI, et al. (2003) Normal thymocyte negative selection in TRAIL-deficient mice. J Exp Med 198: 491496.

40. Lamhamedi-Cherradi SE, Zheng SJ, Maguschak KA, Peschon J, Chen YH (2003) Defective thymocyte apoptosis and accelerated autoimmune diseases in TRAIL-/- mice. Nat Immunol 4: 255-260.

41. Bailey RL, Natividad-Sancho A, Fowler A, Peeling RW, Mabey DC, et al. (2009) Host genetic contribution to the cellular immune response to Chlamydia trachomatis: Heritability estimate from a Gambian twin study. Drugs Today (Barc) 45 Suppl. B: 45-50.

42. Karimi O, Ouburg S, de Vries HJ, Pena AS, Pleijster J, et al. (2009) TLR2 haplotypes in the susceptibility to and severity of Chlamydia trachomatis infections in Dutch women. Drugs Today (Barc) 45 Suppl B: 67-74.

43. den Hartog JE, Lyons JM, Ouburg S, Fennema JS, de Vries HJ, et al. (2009) TLR4 in Chlamydia trachomatis infections: knockout mice, STD patients and women with tubal factor subfertility. Drugs Today (Barc) 45 Suppl B: 75-82.

44. Ouburg S, Lyons JM, Land JA, den Hartog JE, Fennema JS, et al. (2009) TLR9 KO mice, haplotypes and CPG indices in Chlamydia trachomatis infection. Drugs Today (Barc) 45 Suppl B: 83-93.

45. Taylor BD, Darville T, Ferrell RE, Ness RB, Haggerty CL (2013) Racial variation in toll-like receptor variants among women with pelvic inflammatory disease. J Infect Dis 207: 940-946.

46. Taylor BD, Darville T, Ferrell RE, Kammerer CM, Ness RB, et al. (2012) Variants in toll-like receptor 1 and 4 genes are associated with Chlamydia trachomatis among women with pelvic inflammatory disease. J Infect Dis 205: 603-609.

47. Schaefer U, Voloshanenko O, Willen D, Walczak H (2007) TRAIL: a multifunctional cytokine. Front Biosci 12: 3813-3824.

48. Ramsey KH, Sigar IM, Schripsema JH, Denman CJ, Bowlin AK, et al. (2009) Strain and virulence diversity in the mouse pathogen Chlamydia muridarum. Infection and immunity 77: 3284-3293.

49. Byrne GI (2010) Chlamydia trachomatis strains and virulence: rethinking links to infection prevalence and disease severity. The Journal of infectious diseases 201 Suppl 2: S126-133.

50. Pal S, Peterson EM, de la Maza LM (2005) Vaccination with the Chlamydia trachomatis major outer membrane protein can elicit an immune response as 
protective as that resulting from inoculation with live bacteria. Infection and immunity 73: 8153-8160.

51. La Verda D, Byrne GI (1994) Interactions between macrophages and chlamydiae. Immunol Ser 60: 381-399.

52. Azenabor AA, Chaudhry AU (2003) Chlamydia pneumoniae survival in macrophages is regulated by free $\mathrm{Ca} 2+$ dependent reactive nitrogen and oxygen species. The Journal of infection 46: 120-128.

53. Wyrick PB, Brownridge EA (1978) Growth of Chlamydia psittaci in macrophages. Infection and immunity 19: 1054-1060.

54. Walczak H, Degli-Esposti MA, Johnson RS, Smolak PJ, Waugh JY, et al. (1997) TRAIL-R2: a novel apoptosis-mediating receptor for TRAIL. EMBO J 16: 5386-5397.

55. Walczak H, Degli-Esposti MA, Johnson RS, Smolak PJ, Waugh JY, et al. (1997) TRAIL-R2: a novel apoptosis-mediating receptor for TRAIL. The EMBO journal 16: 5386-5397.

56. Eckmann L, Kagnoff MF, Fierer J (1993) Epithelial cells secrete the chemokine interleukin-8 in response to bacterial entry. Infection and immunity 61: 45694574 .

57. Kim SO, Ono K, Tobias PS, Han J (2003) Orphan nuclear receptor Nur77 is involved in caspase-independent macrophage cell death. The Journal of experimental medicine 197: 1441-1452.

58. Cabanski M, Steinmuller M, Marsh LM, Surdziel E, Seeger W, et al. (2008) PKR regulates TLR2/TLR4-dependent signaling in murine alveolar macrophages. American journal of respiratory cell and molecular biology 38: 26-31.

59. Zamanian-Daryoush M, Mogensen TH, DiDonato JA, Williams BR (2000) NFkappaB activation by double-stranded-RNA-activated protein kinase (PKR) is mediated through NF-kappaB-inducing kinase and IkappaB kinase. Molecular and cellular biology 20: 1278-1290.

60. Lu B, Nakamura T, Inouye K, Li J, Tang Y, et al. (2012) Novel role of PKR in inflammasome activation and HMGB1 release. Nature.

61. Hsu LC, Park JM, Zhang K, Luo JL, Maeda S, et al. (2004) The protein kinase PKR is required for macrophage apoptosis after activation of Toll-like receptor 4. Nature 428: 341-345.

62. Sun R, Zhang Y, Lv Q, Liu B, Jin M, et al. (2011) Toll-like receptor 3 (TLR3) induces apoptosis via death receptors and mitochondria by up-regulating the transactivating p63 isoform alpha (TAP63alpha). The Journal of biological chemistry 286: 15918-15928.

63. Ying S, Pettengill M, Ojcius DM, Hacker G (2007) Host-Cell Survival and Death During Chlamydia Infection. Current immunology reviews 3: 31-40.

64. Bannerman DD, Tupper JC, Kelly JD, Winn RK, Harlan JM (2002) The Fasassociated death domain protein suppresses activation of NF-kappa B by LPS and IL-1 beta. The Journal of clinical investigation 109: 419-425.
65. Zhande R, Dauphinee SM, Thomas JA, Yamamoto M, Akira S, et al. (2007) FADD negatively regulates lipopolysaccharide signaling by impairing interleukin-1 receptor-associated kinase 1-MyD88 interaction. Molecular and cellular biology 27: 7394-7404

66. Aliprantis AO, Yang RB, Weiss DS, Godowski P, Zychlinsky A (2000) The apoptotic signaling pathway activated by Toll-like receptor-2. The EMBO journal 19: 3325-3336.

67. Dean D, Kandel RP, Adhikari HK, Hessel T (2008) Multiple Chlamydiaceae species in trachoma: implications for disease pathogenesis and control. PLoS Med 5: e14.

68. Srinivasan T, Bruno WJ, Wan R, Yen A, Duong J, et al. (2012) In vitro recombinants of antibiotic-resistant Chlamydia trachomatis strains have statistically more breakpoints than clinical recombinants for the same sequenced loci and exhibit selection at unexpected loci. J Bacteriol 194: 617-626.

69. Stephens M, Smith NJ, Donnelly P (2001) A new statistical method for haplotype reconstruction from population data. Am J Hum Genet 68: 978-989.

70. Clayton D (2002) A program for estimating frequencies of large haplotypes of SNPs.

71. Atik B, Skwor TA, Kandel RP, Sharma B, Adhikari HK, et al. (2008) Identification of novel single nucleotide polymorphisms in inflammatory genes as risk factors associated with trachomatous trichiasis. PLoS ONE 3: e3600.

72. Abdul-Sater AA, Said-Sadier N, Padilla EV, Ojcius DM (2010) Chlamydial infection of monocytes stimulates IL-1beta secretion through activation of the NLRP3 inflammasome. Microbes and infection / Institut Pasteur 12: 652-661.

73. Perfettini JL, Ojcius DM, Andrews CW Jr, Korsmeyer SJ, Rank RG, et al. (2003) Role of proapoptotic BAX in propagation of Chlamydia muridarum (the mouse pneumonitis strain of Chlamydia trachomatis) and the host inflammatory response. J Biol Chem 278: 9496-9502.

74. Said-Sadier N, Padilla E, Langsley G, Ojcius DM (2010) Aspergillus fumigatus stimulates the NLRP3 inflammasome through a pathway requiring ROS production and the Syk tyrosine kinase. PLoS One 5: e10008.

75. Darville T, O'Neill JM, Andrews CW, Nagarajan UM, Stahl L, et al. (2003) Toll-like receptor-2, but not Toll-like receptor-4, is essential for development of oviduct pathology in chlamydial genital tract infection. J Immunol 171: 61876197.

76. Ramsey KH, Miranpuri GS, Poulsen CE, Marthakis NB, Braune LM, et al. (1998) Inducible nitric oxide synthase does not affect resolution of murine chlamydial genital tract infections or eradication of chlamydiae in primary murine cell culture. Infect Immun 66: 835-838.

77. Welter-Stahl L, Ojcius DM, Viala J, Girardin S, Liu W, et al. (2006) Stimulation of the cytosolic receptor for peptidoglycan, Nod1, by infection with Chlamydia trachomatis or Chlamydia muridarum. Cell Microbiol 8: 1047-1057. 